\title{
A Convenient Route to Diverse Heterocycles through an Addition of $\beta$-Amino Carbonyl Compounds to 3-Halogeno-4-methoxybenzynes
}

\author{
Kyongho Yoon, Sung Min Ha, and Kyongtae Kim*
}

School of Chemistry and Molecular Engineering, Seoul National University, Seoul 151-742, Korea

\section{Content}

\author{
General Procedure \\ $\mathrm{S} 2-\mathrm{S} 3$ \\ Characterizations of Compounds
}

$8 \mathbf{a}$

8b-8d

S4

8e-8g

S5

8h-8i

$8 j-81$

9a'-9b'

10'-12'

13'-15',

$16{ }^{\prime}-17 b^{\prime}$

18'-19'

21'-23a'

23b'-25 
General Procedure for the Reactions of 3-Halogeno-4-methoxy (or 4-hydroxy) benzynes with

$\beta$-amino carbonyl compounds 7, 9-17, 21-22, 2-aminophenyl benzensulfonate (18), bis(2aminophenyl) disulfide (19), phenyl 2-aminobenzensulfonate (20), or 1-amino-2- $(\rho$ tolysulfonamido)-1,2-diphenylethylene (24).

(a) To a solution of substrates $(0.356-0.685 \mathrm{mmol})$ in dried THF $(50 \mathrm{~mL})$ were added 5-(3halogeno-4-methoxyphenyl)thianthrenium perchlorates $(\mathbf{1 b}-\mathbf{e})(0.317-0.571 \mathrm{mmol})$ in portion. This mixture was heated to reflux temperature under an $\mathrm{N}_{2}$ atmosphere. LDA $(0.25 \mathrm{~mL}, 2.0 \mathrm{M}$ in heptane/THF/ethylbenzene) was added with a hypodermic syringe. This addition was repeated 2 or 3 times at intervals of $40-50 \mathrm{~min}$ and the mixture was stirred for $1.5-15 \mathrm{~h}$, depending on the reactions; refer to Table 1. Removal of the solvent in vacuo gave a residue, which was chromatographed on a silica gel column $(20 \times 3 \mathrm{~cm})$. Elution with a mixture of $n$ hexane and EtOAc (2/1, v/v) gave 3 (88 - 97\%), followed by 8, 9'-24', and 4 (5-21\%).

(b) Treament of 5-(3-bromo-4-hydroxyphenyl)thianthenium perchlorate (26) (500 mg, $1.02 \mathrm{mmol})$ with $7 \mathbf{a}(302 \mathrm{mg}, 1.53 \mathrm{mmol})$ in the presence of LDA $(0.25 \mathrm{~mL})$ as described in (a) gave 1bromo-2-hydroxy-9-phenylacridine (25) (210 mg, 59\%), 3 (200 mg, 91\%), and 2-bromo-4(diisopropylamino)phenol (72 mg, 27\%).

(c) Compound $25(86 \%)$ was prepared by treatment of $\mathbf{8 c}(50 \mathrm{mg}, 0.14 \mathrm{mmol})$ with $\mathrm{ZnI}_{2}(90 \mathrm{mg}$, $0.28 \mathrm{mmol})$, followed by addition of TMSI $(112 \mathrm{mg}, 0.561 \mathrm{mmol})$ in $\mathrm{CHCl}_{3}(30 \mathrm{~mL})$ for $48 \mathrm{~h}$ at reflux. 
Synthesis of 4-Fluoro-3-methoxy-10H-phenothiazine 5,5-Dioxide (20') from 4-Fluoro-3-

methoxy-10H-phenothiazine (19'). Sodium perborate tetrahydrate (53 $\mathrm{mg}, 0.345 \mathrm{mmol}$ ) was added portionwise to a stirred solution of the compound 19' (33 mg, $0.134 \mathrm{mmol})$ in glacial acetic acid (20 $\mathrm{mL})$ at $50^{\circ} \mathrm{C}$ for $20 \mathrm{~min}$. Additional stirring was continued at this temperature for $5 \mathrm{~h}$. The solvent was removed in vacuo and the residue was washed with water $(100 \mathrm{~mL})$ to give a crude product 20' (0.101 mmol, 75\%): mp $71-72{ }^{\circ} \mathrm{C}\left(\mathrm{CHCl}_{3} / n\right.$-hexane); ${ }^{1} \mathrm{H}$ NMR $\delta 3.91(\mathrm{~s}, 3 \mathrm{H}), 6.81-7.13(\mathrm{~m}$, $7 \mathrm{H}) ;{ }^{13} \mathrm{C}$ NMR $\delta 56.3,115.5,115.9,118.9,119.0,121.6,127.4,127.9,134.4,135.1,139.5,141.2$, 146.6 ; IR 3473, 3386, 3045, 2348, 1633, 1598, 1587, 1567, 1486, 1455, 1353, 1191, 1166, 1139, $1085,1020,917,858 \mathrm{~cm}^{-1} . \lambda_{\text {max. }}=431 \mathrm{~nm}, \log \varepsilon=4.19$. Anal. calcd for $\mathrm{C}_{13} \mathrm{H}_{10} \mathrm{FNO}_{3} \mathrm{~S}: \mathrm{C}, 55.91 ; \mathrm{H}$, 3.61; N, 5.02; S, 11.48. Found: C, 55.89; H, 3.64; N, 4.97; S, 11.51.

1-Fluoro-2-methoxy-9-phenylacridine (8a): mp $149-150{ }^{\circ} \mathrm{C}\left(\mathrm{CHCl}_{3} / n\right.$-hexane); ${ }^{1} \mathrm{H}$ NMR $\delta$ $4.01(\mathrm{~s}, 3 \mathrm{H}), 7.30-7.43(\mathrm{~m}, 3 \mathrm{H}), 7.51-7.55(\mathrm{~m}, 4 \mathrm{H}), 7.66-7.69(\mathrm{~m}, 2 \mathrm{H}), 8.15(\mathrm{~d}, J=9.3 \mathrm{~Hz}, 1 \mathrm{H})$, $8.20(\mathrm{~d}, J=9.3 \mathrm{~Hz}, 1 \mathrm{H}) ;{ }^{13} \mathrm{C}$ NMR $\delta 55.9,117.7,122.3,123.1,125.6,125.9,126.2,127.3,127.4$, $127.8,128.1,129.0,130.0,131.0,131.7,138,4,146.0,146.2,146.9,153.9$; IR 2931, 1631, 1540, $1471,1443,1395,1261,1216,1096,1040 \mathrm{~cm}^{-1} . \lambda_{\max .}=390 \mathrm{~nm}, \log \varepsilon=2.89$. Anal. calcd for $\mathrm{C}_{20} \mathrm{H}_{14}$ FNO: C, 79.19; H, 4.65; N, 4.62. Found: C, 79.10; H, 4.59; N, 4.61. 
1-Chloro-2-methoxy-9-phenylacridine (8b): mp $151-154{ }^{\circ} \mathrm{C}\left(\mathrm{CHCl}_{3} / n\right.$-hexane); ${ }^{1} \mathrm{H}$ NMR $\delta$ $4.05(\mathrm{~s}, 3 \mathrm{H}), 7.33-7.35(\mathrm{~m}, 3 \mathrm{H}), 7.49-7.55(\mathrm{~m}, 4 \mathrm{H}), 7.65-7.70(\mathrm{~m}, 2 \mathrm{H}), 8.16(\mathrm{~d}, J=9.6 \mathrm{~Hz}, 1 \mathrm{H})$,

$8.33(\mathrm{~d}, J=9.6 \mathrm{~Hz}, 1 \mathrm{H}) ;{ }^{13} \mathrm{C}$ NMR $\delta 56.4,118.7,122.0,123.4,125.3,125.7,126.1,127.0,127.1$, 127.9, 128.0, 129.1, 129.7, 131.1, 131.8, 138,0, 146.2, 146.5, 146.9, 154.0; IR 2946, 1630, 1541, $1474,1441,1340,1265,1221,1096,1040 \mathrm{~cm}^{-1} . \lambda_{\max .}=405 \mathrm{~nm}, \log \varepsilon=3.03$. Anal. calcd for $\mathrm{C}_{20} \mathrm{H}_{14} \mathrm{ClNO}: \mathrm{C}, 75.12 ; \mathrm{H}, 4.41 ; \mathrm{N}, 4.38$. Found: $\mathrm{C}, 74.99 ; \mathrm{H}, 4.45 ; \mathrm{N}, 4.31$.

1-Bromo-2-methoxy-9-phenylacridine (8c): mp $154-155{ }^{\circ} \mathrm{C}\left(\mathrm{CHCl}_{3} / n\right.$-hexane); ${ }^{1} \mathrm{H}$ NMR $\delta$ $4.04(\mathrm{~s}, 3 \mathrm{H}), 7.33-7.41(\mathrm{~m}, 3 \mathrm{H}), 7.51-7.53(\mathrm{~m}, 4 \mathrm{H}), 7.65-7.69(\mathrm{~m}, 2 \mathrm{H}), 8.20(\mathrm{~d}, J=9.4 \mathrm{~Hz}, 1 \mathrm{H})$, $8.34(\mathrm{~d}, J=9.4 \mathrm{~Hz}, 1 \mathrm{H}) ;{ }^{13} \mathrm{C}$ NMR $\delta 57.0,118.8,122.0,123.5,125.8,125.9,126.2,127.0,127.1$, 127.8, 128.0, 129.1, 129.6, 131.1, 131.7, 138.0, 146.0, 146.1, 146.9, 154.4; IR 2940, 1650, 1550, $1471,1443,1340,1261,1214,1096,1044 \mathrm{~cm}^{-1} . \lambda_{\max .}=401 \mathrm{~nm}, \log \varepsilon=2.97$. Anal. calcd for $\mathrm{C}_{20} \mathrm{H}_{14} \mathrm{BrNO}$ : C, 65.95; H, 3.87; N, 3.85. Found: C, 65.90; H, 3.89; N, 3.81.

1-Iodo-2-methoxy-9-phenylacridine (8d): $\mathrm{mp} 154-156{ }^{\circ} \mathrm{C}\left(\mathrm{CHCl}_{3} / n\right.$-hexane); ${ }^{1} \mathrm{H} \mathrm{NMR} \delta 4.03$ $(\mathrm{s}, 3 \mathrm{H}), 7.35-7.37(\mathrm{~m}, 3 \mathrm{H}), 7.55-7.61(\mathrm{~m}, 4 \mathrm{H}), 7.81-7.83(\mathrm{~m}, 2 \mathrm{H}), 8.20(\mathrm{~d}, J=8.9 \mathrm{~Hz}, 1 \mathrm{H}), 8.34$ $(\mathrm{d}, J=8.9 \mathrm{~Hz}, 1 \mathrm{H}) ;{ }^{13} \mathrm{C}$ NMR $\delta 57.6,120.1,122.3,123.1,125.1,125.6,125.9,127.3,127.6,128.0$, 128.0, 129.0, 129.6, 131.3, 131.6, 138.4, 146.0, 146.4, 147.1, 155.1; IR 2938, 1631, 1536, 1474, 
$1445,1341,1266,1218,1096,1041 \mathrm{~cm}^{-1} . \lambda_{\max .}=403 \mathrm{~nm}, \log \varepsilon=3.17$. Anal. calcd for $\mathrm{C}_{20} \mathrm{H}_{14} \mathrm{INO}$ :

C, 58.41; H, 3.43; N, 3.41. Found: C, 58.50; H, 3.49; N, 3.39.

5-Chloro-1-fluoro-2-methoxy-7-methyl-9-phenylacridine (8e): mp $143-144{ }^{\circ} \mathrm{C}\left(\mathrm{CHCl}_{3} / n\right.$ hexane); ${ }^{1} \mathrm{H}$ NMR $\delta 2.51(\mathrm{~s}, 3 \mathrm{H}), 3.95(\mathrm{~s}, 3 \mathrm{H}), 7.47-7.50(\mathrm{~m}, 2 \mathrm{H}), 7.55(\mathrm{~d}, J=7.93 \mathrm{~Hz}, 1 \mathrm{H}), 7.61-$ $7.66(\mathrm{~m}, 2 \mathrm{H}), 7.73-7.79(\mathrm{~d}, J=7.91,2 \mathrm{H}) ;{ }^{13} \mathrm{C}$ NMR $\delta 16.5,55.4,116.7,122.1,123.9,126.0,126.8$, 127.1, 127.8, 128.4, 129.1, 129.6, 131.1, 131.8, 137.1, 138,0, 146.2, 146.5, 146.9, 154.1; IR 2928, 1644, 1540, 1472, 1433, 1399, 1340, 1265, 1212, $1097 \mathrm{~cm}^{-1} . \lambda_{\max .}=457 \mathrm{~nm}, \log \varepsilon=3.52$. Anal. calcd for $\mathrm{C}_{20} \mathrm{H}_{13}$ ClFNO: C, 71.12; H, 3.88; N, 4.15. Found: C, 71.10; H, 3.81; N, 4.11.

7-Chloro-1-fluoro-2-methoxy-9-phenylacridine (8f): mp $144-147{ }^{\circ} \mathrm{C}\left(\mathrm{CHCl}_{3} / n\right.$-hexane); ${ }^{1} \mathrm{H}$ NMR $\delta 4.00(\mathrm{~s}, 3 \mathrm{H}), 7.34-7.35(\mathrm{~m} \mathrm{2H}), 7.47-7.70(\mathrm{~m}, 6 \mathrm{H}), 7.76(\mathrm{~d}, J=7.91,1 \mathrm{H}), 8.08(\mathrm{~d}, \mathrm{~J}=$ $9.91 \mathrm{~Hz}, 1 \mathrm{H}) ;{ }^{13} \mathrm{C}$ NMR $\delta 16.5,55.4,116.7,123.9,126.0,126.8,127.1,127.8,128.4,129.1,129.6$, 131.1, 131.8, 137.1, 138,0, 146.2, 146.5, 146.9, 154.1, 156.3; IR 2940, 1630, 1536, 1474, 1440, 1341, 1267, 1220, 1096, $1040 \mathrm{~cm}^{-1} . \lambda_{\max .}=411 \mathrm{~nm}, \log \varepsilon=3.11$. Anal. calcd for $\mathrm{C}_{20} \mathrm{H}_{13} \mathrm{ClFNO}: \mathrm{C}$, 71.12; H, 3.88; N, 4.15. Found: C, 71.08; H, 3.85; N, 4.17.

1,7-Dichloro-2-methoxy-9-phenylacridine (8g): mp 139 - $141{ }^{\circ} \mathrm{C}\left(\mathrm{CHCl}_{3} / n\right.$-hexane); ${ }^{1} \mathrm{H} \mathrm{NMR} \delta$ $3.73(\mathrm{~s}, 3 \mathrm{H}), 7.22-7.48(\mathrm{~m}, 6 \mathrm{H}), 7.62-8.00(\mathrm{~m}, 4 \mathrm{H}) ;{ }^{13} \mathrm{C}$ NMR $\delta 55.5,109.3,119.8,121.9,122.3$, 
$126.2,126.8,126.9,129.0,129.8,130.0,130.7,132.0,132.5,138.1,140.1,146.0,147.1,150.6$, 158.3; IR 2980, 1655, 1631, 1530, 1475, 1442, 1440, 1340, 1267, 1221, 1095, 1040, $982 \mathrm{~cm}^{-1} . \lambda_{\max }$ $=397 \mathrm{~nm}, \log \varepsilon=3.03$. Anal. calcd for $\mathrm{C}_{20} \mathrm{H}_{13} \mathrm{Cl}_{2} \mathrm{NO}$ : C, 67.81; H, 3.70; N, 3.95. Found: C, 67.73; H, 3.65; N, 4.02 .

1-Bromo-7-chloro-2-methoxy-9-phenylacridine (8h): mp $138-139{ }^{\circ} \mathrm{C}\left(\mathrm{CHCl}_{3} / n\right.$-hexane); ${ }^{1} \mathrm{H}$ NMR $\delta 3.80(\mathrm{~s}, 3 \mathrm{H}), 6.98-7.23(\mathrm{~m}, 6 \mathrm{H}), 7.61-7.89(\mathrm{~m}, 4 \mathrm{H}) ;{ }^{13} \mathrm{C}$ NMR $\delta 55.6,110.1,119.0$, $120.5,121.9,126.2,126.5,126.9,129.3,129.5,130.0,130.3,131.3,132.5,137.9,140.0,146.3$, 147.2, 150.6, 157.9; IR 2898, 1650, 1645, 1531, 1475, 1443, 1344, 1280, 1220, 1117, 1094, 1041, $988 \mathrm{~cm}^{-1} . \lambda_{\text {max. }}=388 \mathrm{~nm}, \log \varepsilon=2.77$. Anal. calcd for $\mathrm{C}_{20} \mathrm{H}_{13} \mathrm{BrClNO}: \mathrm{C}, 60.25 ; \mathrm{H}, 3.29 ; \mathrm{N}, 3.51$. Found: C, 60.21; H, 3.25; N, 3.57.

7-Chloro-1-iodo-2-methoxy-9-phenylacridine (8i): mp $135-138{ }^{\circ} \mathrm{C}\left(\mathrm{CHCl}_{3} / n\right.$-hexane); ${ }^{1} \mathrm{H}$ NMR $\delta 3.91(\mathrm{~s}, 3 \mathrm{H}), 7.02-7.23(\mathrm{~m}, 6 \mathrm{H}), 7.60-7.93(\mathrm{~m}, 4 \mathrm{H}) ;{ }^{13} \mathrm{C}$ NMR $\delta 55.8,110.0,118.5$, $119.7,120.9,125.9,126.3,126.8,129.3,129.5,130.2,130.5,131.3,132.7,137.9,140.4,146.3$, 147.1, 150.4, 158.1; IR 2995, 2890, 1651, 1534, 1480, 1444, 1441, 1345, 1298, 1221, 1215, 1095, $1043,1022,980 \mathrm{~cm}^{-1} . \lambda_{\text {max. }}=401 \mathrm{~nm}, \log \varepsilon=3.01$. Anal. calcd for $\mathrm{C}_{20} \mathrm{H}_{13}$ ClINO: $\mathrm{C}, 53.90 ; \mathrm{H}, 2.94$; N, 3.14; O, 3.59. Found: C, 53.91; H, 2.95; N, 3.20; O, 3.50. 
1-Fluoro-2-methoxy-7-methyl-9-phenylacridine (8j): mp $143-144{ }^{\circ} \mathrm{C}\left(\mathrm{CHCl}_{3} / n\right.$-hexane); ${ }^{1} \mathrm{H}$

NMR $\delta 2.55(\mathrm{~s}, 3 \mathrm{H}), 3.95(\mathrm{~s}, 3 \mathrm{H}), 7.33(\mathrm{~d}, J=7.99,1 \mathrm{H}), 7.47-7.50(\mathrm{~m}, 2 \mathrm{H}), 7.55(\mathrm{~d}, J=7.93 \mathrm{~Hz}$, 1H), $7.61-7.66(\mathrm{~m}, 2 \mathrm{H}), 7.73-7.79(\mathrm{~d}, J=7.91,2 \mathrm{H}), 8.07-8.09(\mathrm{~m}, 2 \mathrm{H}) ;{ }^{13} \mathrm{C}$ NMR $\delta 16.5,55.4$, $116.7,122.0,123.9,125.6,126.0,126.8,127.1,127.8,128.4,129.1,129.6,131.1,131.8,137.1$, 138,0, 146.2, 146.5, 146.9, 154.1; IR 2928, 1644, 1540, 1472, 1433, 1399, 1340, 1265, 1212, 1097 $\mathrm{cm}^{-1} . \lambda_{\text {max. }}=399 \mathrm{~nm}, \log \varepsilon=2.81$. Anal. calcd for $\mathrm{C}_{21} \mathrm{H}_{16} \mathrm{FNO}: \mathrm{C}, 79.48 ; \mathrm{H}, 5.08 ; \mathrm{N}, 4.41$. Found: $\mathrm{C}$, $79.51 ; \mathrm{H}, 5.11 ; \mathrm{N}, 4.47$.

1-Bromo-9-(3-chlorophenyl)-2-methoxyacridine (8k): mp $139-142{ }^{\circ} \mathrm{C}\left(\mathrm{CHCl}_{3} / n\right.$-hexane); ${ }^{1} \mathrm{H}$ NMR $\delta 3.98$ (s, 3H), $7.14-7.58(\mathrm{~m}, 6 \mathrm{H}), 7.76$ - $8.01(\mathrm{~m}, 4 \mathrm{H}) ;{ }^{13} \mathrm{C}$ NMR $\delta 55.5,118.1,120.9,123.1$, $125.7,126.4,126.9,127.2,127.8,129.0,129.3,129.7,130.5,131.8,137.5,138.0,146.1,146.5$, 146.9, 154.1; IR 2940, 1630, 1536, 1474, 1440, 1341, 1267, 1220, 1096, $1040 \mathrm{~cm}^{-1} . \lambda_{\max .}=406 \mathrm{~nm}$, $\log \varepsilon=2.93$. Anal. calcd for $\mathrm{C}_{20} \mathrm{H}_{13}$ BrClNO: $\mathrm{C}, 60.25 ; \mathrm{H}, 3.29 ; \mathrm{N}, 3.51$. Found: $\mathrm{C}, 60.21 ; \mathrm{H}, 3.35$; $\mathrm{N}, 3.57$.

7-Chloro-9-(2-chlorophenyl)-1-fluoro-2-methoxyacridine (81): mp $144-145{ }^{\circ} \mathrm{C}\left(\mathrm{CHCl}_{3} / n\right.$ hexane); ${ }^{1} \mathrm{H}$ NMR $\delta 4.00(\mathrm{~s}, 3 \mathrm{H}), 7.34-7.57(\mathrm{~m} 6 \mathrm{H}), 7.70-7.91(\mathrm{~m}, 3 \mathrm{H}) ;{ }^{13} \mathrm{C} \mathrm{NMR}(75 \mathrm{MHz}$, $\left.\mathrm{CDCl}_{3}\right) \delta 56.0,118.9,121.6,122.8,125.1,126.7,126.8,127.0,127.4,128.2,129.1,130.0,131.5$, 131.8, 137.0, 138.0, 146.4, 146.7, 147.3, 155.5; IR (KBr) 3011, 2995, 1635, 1621, 1554, 1447, 1340, 
$1280,1225,1220,1098,1044 \mathrm{~cm}^{-1} . \lambda_{\text {max. }}=410 \mathrm{~nm}, \log \varepsilon=2.94$. Anal. calcd for $\mathrm{C}_{20} \mathrm{H}_{12} \mathrm{Cl}_{2} \mathrm{FNO}: \mathrm{C}$,

$64.54 ; \mathrm{H}, 3.25 ; \mathrm{N}, 3.76$. Found: C, 64.51; H, 3.25; N, 3.77.

5'-Aza-1'-fluoro-2'-methoxynaphtho[3,2,1-de]anthracen-9-one $\quad$ (9a'): mp $160-163 \quad{ }^{\circ} \mathrm{C}$ $\left(\mathrm{CH}_{3} \mathrm{CN}\right) ;{ }^{1} \mathrm{H}$ NMR $\delta 3.90(\mathrm{~s}, 3 \mathrm{H}), 6.83-6.90(\mathrm{~m}, 2 \mathrm{H}), 7.15-7.16(\mathrm{~m}, 1 \mathrm{H}), 7.51(\mathrm{~d}, J=7.71 \mathrm{~Hz}$, 1H), $7.77-7.81(\mathrm{~m}, 2 \mathrm{H}), 7.93(\mathrm{~d}, J=7.71,1 \mathrm{H}), 8.27-8.31(\mathrm{~m}, 2 \mathrm{H}) ;{ }^{13} \mathrm{C}$ NMR $\delta 54.1,110.3,121.7$, $126.5,126.8,128.1,130.2,130.5,130.8,131.4,132.5,133.2,134.2,136.0,136.6,139.7,140.2$, 141.2, 145.9, 151.0, 186.1; IR 2940, 1635, 1555, 1471, 1454, 1441, 1339, 1261, 1221, 1096, 1040 $\mathrm{cm}^{-1} . \lambda_{\max .}=521 \mathrm{~nm}, \log \varepsilon=6.89$. Anal. calcd for $\mathrm{C}_{21} \mathrm{H}_{12} \mathrm{FNO}_{2}: \mathrm{C}, 76.59 ; \mathrm{H}, 3.67 ; \mathrm{N}, 4.25$. Found: C, $76.51 ; \mathrm{H}, 3.65 ; \mathrm{N}, 4.27$.

5'-Aza-1'-fluoro-2'-methoxy-3-methylnaphtho[3,2,1-de]anthracen-9-one (9b'): mp 162 - 164

${ }^{\circ} \mathrm{C}\left(\mathrm{CH}_{3} \mathrm{CN}\right) ;{ }^{1} \mathrm{H}$ NMR $\delta 2.06(\mathrm{~s}, 3 \mathrm{H}), 3.91(\mathrm{~s}, 3 \mathrm{H}), 6.83-6.90(\mathrm{~m}, 2 \mathrm{H}), 7.15-7.16(\mathrm{~m}, 1 \mathrm{H}), 7.51$ $(\mathrm{d}, J=7.71 \mathrm{~Hz}, 1 \mathrm{H}), 7.77-7.81(\mathrm{~m}, 2 \mathrm{H}), 7.93(\mathrm{~d}, J=7.71,1 \mathrm{H}), 8.27-8.31(\mathrm{~m}, 1 \mathrm{H}) ;{ }^{13} \mathrm{C} \mathrm{NMR} \delta$ $16.4,55.3,110.1,122.2,126.2,126.6,128.7,130.2,130.6,130.8,131.4,132.7,133.0,134.2,136.1$, 136.2, 139.7, 140.4, 141.2, 145.9, 150.9, 187.0; IR (KBr) 2941, 1631, 1555, 1474, 1455, 1441, 1339, $1261,1221,1096,1045 \mathrm{~cm}^{-1} . \lambda_{\max }=489 \mathrm{~nm}, \log \varepsilon=6.31$. Anal. calcd for $\mathrm{C}_{22} \mathrm{H}_{14} \mathrm{FNO}_{2}: \mathrm{C}, 76.96$; H, 4.11; N, 4.08. Found: C, 77.01; H, 4.15; N, 4.07. 
Cyclohexyl[1,2,3-kl;4,5,6-k'l']-5,13-diamino-4,12-difluoro-3,11-dimethoxydiacridine (10'): mp

$165-167{ }^{\circ} \mathrm{C}\left(\mathrm{CHCl}_{3} / n\right.$-hexane); ${ }^{1} \mathrm{H}$ NMR $\delta 3.73$ (s, 6H), 4.0 (br s, 4H), $6.84(\mathrm{~d}, J=7.89,2 \mathrm{H}), 7.23$

$-7.31(\mathrm{~m}, 4 \mathrm{H}), 7.75(\mathrm{~d}, J=7.89,2 \mathrm{H}) ;{ }^{13} \mathrm{C}$ NMR $\delta 56.1,111.2,116.4,120.8,121.1,122.2,122.7$,

125.1, 126.9, 131.7, 136.9, 142.0, 144.8, 150.5; IR 3473, 3386, 3045, 2940, 1631, 1475, 1455, 1265,

$1220,1091,1046 \mathrm{~cm}^{-1} . \mathrm{MS}(\mathrm{m} / \mathrm{z}, 100 \%) 480\left(\mathrm{M}^{+}, 100\right) . \lambda_{\max .}=470 \mathrm{~nm}, \log \varepsilon=5.91$. Anal. calcd for $\mathrm{C}_{28} \mathrm{H}_{18} \mathrm{~F}_{2} \mathrm{~N}_{4} \mathrm{O}_{2}$ : C, 69.99; H, 3.78; N, 11.66. Found: C, 70.03; H, 3.75; N, 11.73.

1-Fluoro-2,6,7-trimethoxyacridin-9-one (11'): mp $141-143{ }^{\circ} \mathrm{C}\left(\mathrm{CH}_{3} \mathrm{CN}\right) ;{ }^{1} \mathrm{H}$ NMR $\delta 3.70$ (s, 3H), $3.72(\mathrm{~s}, 6 \mathrm{H}), 5.12(\mathrm{~s}, 1 \mathrm{H}), 7.31(\mathrm{~d}, J=7.93,1 \mathrm{H}), 7.33(\mathrm{~d}, J=7.93,1 \mathrm{H}), 7.58(\mathrm{~s}, 1 \mathrm{H}), 7.78(\mathrm{~s}$, $1 \mathrm{H}) ;{ }^{13} \mathrm{C}$ NMR $\delta 55.8,56.3,100.3,101.9,108.1,115.1,122.5,126.8,136.9,142.0,145.3,148.6$, 151.0, 154.5, 157.7, 187.3; IR 2945, 1631, 1554, 1550, 1477, 1454, 1441, 1265, 1221, $1095 \mathrm{~cm}^{-1}$. $\lambda_{\text {max. }}=421 \mathrm{~nm}, \log \varepsilon=3.74$. Anal. calcd for $\mathrm{C}_{16} \mathrm{H}_{14} \mathrm{FNO}_{4}: \mathrm{C}, 63.36 ; \mathrm{H}, 4.65 ; \mathrm{N}, 4.62$. Found: $\mathrm{C}$, 63.31; H, 4.66; N, 4.67.

9-Fluoro-8-methoxy-5H-pyrazino[2,3-b]quinolin-10-one (12'): mp $167-168{ }^{\circ} \mathrm{C}\left(\mathrm{CH}_{3} \mathrm{CN}\right) ;{ }^{1} \mathrm{H}$ NMR $\delta 3.96(\mathrm{~s}, 3 \mathrm{H}), 6.25$ (br s, 1H), $6.52-6.68(\mathrm{~m}, 2 \mathrm{H}), 7.15-7.63(\mathrm{~m}, 2 \mathrm{H}) ;{ }^{13} \mathrm{C}$ NMR $\delta 56.3$, $110.4,114.8,122.7,126.5,130.3,142.3,144.8,144.9,146.9,149.1,187.0$; IR 3440, 2928, 1651, $1470,1433,1400,1353,1261,1222,1097 \mathrm{~cm}^{-1} . \lambda_{\max .}=451 \mathrm{~nm}, \log \varepsilon=5.11$. Anal. calcd for $\mathrm{C}_{12} \mathrm{H}_{8} \mathrm{FN}_{3} \mathrm{O}_{2}:$ C, 58.78; H, 3.29; N, 17.14. Found: C, 58.81; H, 3.26; N, 17.17. 
4,9-Dihydro-5-fluoro-6-methoxy-1-phenyl-1H-pyrazolo[3,2-b]quinolin-4-one (13'): mp 136 -

$139{ }^{\circ} \mathrm{C}\left(\mathrm{CHCl}_{3} / n\right.$-hexane); ${ }^{1} \mathrm{H}$ NMR $\delta 3.80(\mathrm{~s}, 3 \mathrm{H}), 6.46(\mathrm{~d}, J=7.93,2 \mathrm{H}), 6.62(\mathrm{t}, J=7.93,2 \mathrm{H})$,

$7.01(\mathrm{~d}, J=7.93,2 \mathrm{H}), 7.29(\mathrm{~d}, J=7.89,1 \mathrm{H}), 7.33(\mathrm{~d}, J=7.89,1 \mathrm{H}), 7.72(\mathrm{~s}, 2 \mathrm{H}) ;{ }^{13} \mathrm{C}$ NMR $\delta 55.7$, $118.6,118.8,121.5,122.5,126.0,127.2,128.3,129.1,132.8,139.7,140.7,141.5,143.0,145.4$, 150.8, 155.1, 187.0; IR 3472, 3040, 2945, 2358, 1633, 1598, 1587, 1567, 1486, 1455, 1353, 1190, $1166,1139,1085,1020,917,858 \mathrm{~cm}^{-1} \cdot \lambda_{\text {max. }}=477 \mathrm{~nm}, \log \varepsilon=5.65$. Anal. calcd for $\mathrm{C}_{18} \mathrm{H}_{13} \mathrm{FN}_{2} \mathrm{O}_{2}$ : C, 70.12; H, 4.25; N, 9.09. Found: C, 70.21; H, 4.26; N, 9.14.

4,9-Dihydro-8-fluoro-7-methoxythieno[3,2-b]quinolin-9-one (14'): mp $140-141{ }^{\circ} \mathrm{C}\left(\mathrm{CHCl}_{3} /\right.$ $n$-hexane); ${ }^{1} \mathrm{H}$ NMR $\delta 3.73$ (s, 3H), 5.01 (br s, 1H), 6.96 - $7.10(\mathrm{~m}, 2 \mathrm{H}), 7.34(\mathrm{~d}, J=7.91,1 \mathrm{H}), 7.76$ $(\mathrm{d}, J=7.91,1 \mathrm{H}) ;{ }^{13} \mathrm{C}$ NMR $\delta 56.1,110.4,121.7,125.4,126.5,126.7,130.3,141.3,146.5,149.1$, 150.7, 187.7; IR 3440, 3100, 2928, 1650, 1540, 1471, 1433, 1405, 1347, 1260, 1212, 1098, $935 \mathrm{~cm}^{-}$ 1. $\lambda_{\text {max. }}=436 \mathrm{~nm}, \log \varepsilon=4.99$. Anal. calcd for $\mathrm{C}_{12} \mathrm{H}_{8} \mathrm{FNO}_{2} \mathrm{~S}: \mathrm{C}, 57.82 ; \mathrm{H}, 3.23 ; \mathrm{N}, 5.62 ; \mathrm{S}, 12.86$. Found: C, 57.81; H, 3.26; N, 5.67; S, 12.82 .

2,9-Dihydro-5-fluoro-6-methoxypyrazolo[3,4-b]quinolin-4-one (15'): mp $141-144{ }^{\circ} \mathrm{C}\left(\mathrm{CHCl}_{3} /\right.$ $\mathrm{MeOH}) ;{ }^{1} \mathrm{H}$ NMR $\delta 3.88(\mathrm{~s}, 3 \mathrm{H}), 5.01(\mathrm{br} \mathrm{s}, 1 \mathrm{H}), 6.68-6.81(\mathrm{~m}, 2 \mathrm{H}), 7.61(\mathrm{~d}, J=7.91 \mathrm{~Hz}, 1 \mathrm{H})$, 10.8 (br s, $1 \mathrm{H}) ;{ }^{13} \mathrm{C}$ NMR $\delta 56.2,111.8,117.8,125.4,132.0,133.5,139.1,141.6,149.5,154.0$, 
187.3; IR 3100, 2928, 1735, 1640, 1541, 1477, 1470, 1433, 1400, 1340, 1265, 1211, $1087 \mathrm{~cm}^{-1} . \lambda_{\max }$. $=550 \mathrm{~nm}, \log \varepsilon=7.02$. Anal. calcd for $\mathrm{C}_{11} \mathrm{H}_{8} \mathrm{FN}_{3} \mathrm{O}_{2}: \mathrm{C}, 56.65 ; \mathrm{H}, 3.46 ; \mathrm{N}, 18.02$. Found: C, 56.71; H, 3.47; N, 17.97.

1-Fluoro-2-methoxyacridin-9-ylamine (16'): mp 129 - $130{ }^{\circ} \mathrm{C}\left(\mathrm{CHCl}_{3} / n\right.$-hexane); ${ }^{1} \mathrm{H}$ NMR $\left(\mathrm{DMSO}-d_{6}\right) \delta 3.83(\mathrm{~s}, 3 \mathrm{H}), 5.71(\mathrm{~s}, 2 \mathrm{H}), 7.73-7.85(\mathrm{~m}, 4 \mathrm{H}), 7.91-7.94(\mathrm{~m}, 2 \mathrm{H}) ;{ }^{13} \mathrm{C}$ NMR $\left(\mathrm{DMSO}-d_{6}\right) \delta 56.0,104.3,117.8,120.3,122.2,125.0,126.9,128.9,129.5,136.9,142.0,148.7$, 149.7, 150.3; IR 3453, 3357, 3093, 2996, 2952, 2836, 1673, 1623, 1598, 1517, 1411, 1309, 1214, $1002,952,858 \mathrm{~cm}^{-1} . \lambda_{\text {max. }}=450 \mathrm{~nm}, \log \varepsilon=4.19\left(\mathrm{CH}_{3} \mathrm{CN}\right)$. Anal. calcd for $\mathrm{C}_{14} \mathrm{H}_{11} \mathrm{FN}_{2} \mathrm{O}: \mathrm{C}, 69.41$; H, 4.58; N, 11.56. Found: C, 69.45; H, 4.52; N, 11.57.

1-Fluoro-2-methoxy-9-methylacridine (17a'): mp $153-154{ }^{\circ} \mathrm{C}\left(\mathrm{CH}_{3} \mathrm{CN}\right) ;{ }^{1} \mathrm{H}$ NMR $\delta 2.37$ (s, 3H), $3.75(\mathrm{~s}, 3 \mathrm{H}), 7.31(\mathrm{~d}, J=7.90,1 \mathrm{H}), 7.42(\mathrm{~d}, J=7.90,1 \mathrm{H}), 7.58-7.78(\mathrm{~m}, 4 \mathrm{H}) ;{ }^{13} \mathrm{C}$ NMR $\delta$ $17.5,56.0,110.7,121.5,122.0,123.4,125.6,126.7,128.2,129.5,136.9,141.0,142.3,14.9,150.6$; IR $2928,1644,1540,1472,1433,1261,1221,1096,1040 \mathrm{~cm}^{-1} \cdot \lambda_{\max .}=402 \mathrm{~nm}, \log \varepsilon=3.24$. Anal. calcd for $\mathrm{C}_{15} \mathrm{H}_{12}$ FNO: C, 74.67; H, 5.01; N, 5.81. Found: C, 74.72; H, 5.07; N, 5.83.

1-Fluoro-9-methyl-2,6,7-trimethoxyacridine (17b'): mp $141-143{ }^{\circ} \mathrm{C}\left(\mathrm{CH}_{3} \mathrm{CN}\right) ;{ }^{1} \mathrm{H} \mathrm{NMR} \delta 3.70$ (s, 3H), $3.72(\mathrm{~s}, 6 \mathrm{H}), 7.31(\mathrm{~d}, J=7.93,1 \mathrm{H}), 7.33(\mathrm{~d}, J=7.93,1 \mathrm{H}), 7.58(\mathrm{~s}, 1 \mathrm{H}), 7.78-7.81(\mathrm{~m}, 2 \mathrm{H})$; 
${ }^{13} \mathrm{C}$ NMR $\delta 55.8,56.3,100.3,101.9,108.1,115.1,122.5,126.8,136.9,142.0,145.3,148.6,151.0$, 154.5, 157.7; IR (KBr) 3345, 3050, 2996, 1635, 1621, 1590, 1415, 1309, 1207, 950, $891 \mathrm{~cm}^{-1} . \lambda_{\max }$. $=419 \mathrm{~nm}, \log \varepsilon=3.67$. Anal. calcd for $\mathrm{C}_{16} \mathrm{H}_{14} \mathrm{FNO}_{4}: \mathrm{C}, 63.36 ; \mathrm{H}, 4.65 ; \mathrm{N}, 4.62$. Found: $\mathrm{C}, 63.32 ; \mathrm{H}$, $4.67 ; \mathrm{N}, 4.59$.

4-Fluoro-3-methoxy-10H-phenoxazine (18'): mp 132 - $135{ }^{\circ} \mathrm{C}\left(\mathrm{CHCl}_{3} / n\right.$-hexane); ${ }^{1} \mathrm{H}$ NMR $\delta$ $3.90(\mathrm{~s}, 3 \mathrm{H}), 5.01(\mathrm{~s}, 1 \mathrm{H}), 6.69-6.71(\mathrm{~m}, 2 \mathrm{H}), 6.78-6.99(\mathrm{~m}, 4 \mathrm{H}) ;{ }^{13} \mathrm{C} \mathrm{NMR} \delta 56.5,109.7,114.2$, $117.7,117.8,120.2,122.5,125.9,132.0,133.5,137.4,138.2,145.5$; IR 3440, 3100, 2998, 1650, $1541,1480,1433,1343,1261,1212,1098 \mathrm{~cm}^{-1} . \lambda_{\max .}=455 \mathrm{~nm}, \log \varepsilon=4.02$. Anal. calcd for $\mathrm{C}_{13} \mathrm{H}_{10} \mathrm{FNO}_{2}$ : C, 67.53; H, 4.36; N, 6.06. Found: C, 67.52; H, 4.37; N, 6.03.

4-Fluoro-3-methoxy-10H-phenothiazine (19'): $\mathrm{mp} 131-133{ }^{\circ} \mathrm{C}\left(\mathrm{CHCl}_{3} / n\right.$-hexane); ${ }^{1} \mathrm{H} \mathrm{NMR} \delta$ $3.90(\mathrm{~s}, 3 \mathrm{H}), 4.99($ br s, $1 \mathrm{H}), 6.69-6.72(\mathrm{~m}, 2 \mathrm{H}), 6.91-7.23(\mathrm{~m}, 4 \mathrm{H}) ;{ }^{13} \mathrm{C}$ NMR $\delta 56.1,110.2$, $115.1,115.2,120.9,121.2,122.1,127.9,133.5,138.5,139.1,145.7,151.1$; IR 3473, 3340, 3011, $2998,2348,1566,1485,1455,1212,1191,1085 \mathrm{~cm}^{-1} \cdot \lambda_{\max .}=415 \mathrm{~nm}, \log \varepsilon=3.97$. Anal. calcd for $\mathrm{C}_{13} \mathrm{H}_{10}$ FNOS: C, 63.14; H, 4.08; N, 5.66; O, 6.47; S, 12.97. Found: C, 63.22; H, 4.14; N, 5.71; O, 6.77; S, 12.91 . 
1,2-Dihydro-11-fluoro-10-methoxy-1,2,7-triazabenzo[de] anthracen-3-one (21'): mp 132 - 134

${ }^{\circ} \mathrm{C}\left(\mathrm{CHCl}_{3} / n\right.$-hexane); ${ }^{1} \mathrm{H}$ NMR $\delta 3.73(\mathrm{~s}, 3 \mathrm{H}), 4.01$ (br s, $\left.1 \mathrm{H}\right), 7.31(\mathrm{~d}, J=7.99,1 \mathrm{H}), 7.55(\mathrm{~d}, J=$ 7.99, 1H), $7.69-7.86(\mathrm{~m}, 2 \mathrm{H}), 8.05($ br s, $1 \mathrm{H}), 8.22(\mathrm{~d}, J=7.91 \mathrm{~Hz}, 1 \mathrm{H}) ;{ }^{13} \mathrm{C}$ NMR $\delta 56.8,110.9$, $114.3,122.5,125.9,126.4,128.9,130.0,135.5,136.9,141.7,142.0,147.2,151.3,167.3$; IR 2928, $1735,1644,1540,1472,1433,1399,1340,1265,1212,1097 \mathrm{~cm}^{-1} \cdot \lambda_{\max .}=549 \mathrm{~nm}, \log \varepsilon=7.23$. Anal. calcd for $\mathrm{C}_{15} \mathrm{H}_{10} \mathrm{FN}_{3} \mathrm{O}_{2}$ : C, 63.60; H, 3.56; N, 14.83. Found: C, 63.69; H, 3.54; N, 14.91 .

6-Aza-10-fluoro-9-methoxy-5-oxanaphthacen-12-one (22'): mp $140-143{ }^{\circ} \mathrm{C}\left(\mathrm{CHCl}_{3} / n\right.$ hexane); ${ }^{1} \mathrm{H}$ NMR $\delta 3.73(\mathrm{~s}, 3 \mathrm{H}), 6.92-7.01(\mathrm{~m}, 2 \mathrm{H}), 7.29(\mathrm{~d}, J=7.89,1 \mathrm{H}), 7.37(\mathrm{~m}, 1 \mathrm{H}), 7.61(\mathrm{~d}, J$ $=7.89,1 \mathrm{H}), 7.64(\mathrm{~m}, 1 \mathrm{H}), 8.42(\mathrm{~s}, 1 \mathrm{H}) ;{ }^{13} \mathrm{C} \mathrm{NMR} \delta 56.0,116.8,121.2,122.0,124.5,124.8,125.0$, $125.8,130.5,131.1,133.4,138.0,140.3,145.5,155.3,174.3,187.0$; IR 2928, 1775, 1655, 1547, $1475,1430,1400,1347,1262,1211,1097 \mathrm{~cm}^{-1} \cdot \lambda_{\max .}=540 \mathrm{~nm}, \log \varepsilon=6.46$. Anal. calcd for $\mathrm{C}_{15} \mathrm{H}_{10} \mathrm{FN}_{3} \mathrm{O}_{2}$ : C, 63.60; H, 3.56; N, 14.83. Found: C, 63.69; H, 3.54; N, 14.91.

5-Fluoro-6-methoxy-2-methylquinolin-4-one (23a'): mp $134-137{ }^{\circ} \mathrm{C}\left(\mathrm{CH}_{3} \mathrm{CN}\right) ;{ }^{1} \mathrm{H}$ NMR $\delta$ $3.23(\mathrm{~s}, 3 \mathrm{H}), 3.88(\mathrm{~s}, 3 \mathrm{H}), 7.20-7.31(\mathrm{~m}, 2 \mathrm{H}), 7.32(\mathrm{~s}, 1 \mathrm{H}) ;{ }^{13} \mathrm{C}$ NMR $\delta$ 21.6, 56.5, 106.8, 108.4, 122.5, 125.9, 129.8, 141.2, 146.8, 150.1, 158.4; IR 3296, 2912, 2208, 1616, 1568, 1488, 1459, 1276, $1248,1212,1177,1136,1043,1017,829,809,777 \mathrm{~cm}^{-1} \cdot \lambda_{\max .}=490 \mathrm{~nm}, \log \varepsilon=4.05$. Anal. calcd for $\mathrm{C}_{11} \mathrm{H}_{10} \mathrm{FNO}_{2}$ : C, 63.76; H, 4.86; N, 6.76. Found: C, 63.79; H, 4.79; N, 6.76. 
5-Fluoro-6-methoxy-2-trifluoromethylquinolin-4-one (23b'): mp $136-138{ }^{\circ} \mathrm{C}\left(\mathrm{CHCl}_{3} / n\right.$ hexane); ${ }^{1} \mathrm{H}$ NMR $\delta 3.88$ (s, 3H), $7.21-7.30(\mathrm{~m}, 2 \mathrm{H}), 7.33(\mathrm{~s}, 1 \mathrm{H}) ;{ }^{13} \mathrm{C}$ NMR $\delta 56.1,100.6,111.7$, 120.0, 124.2, 127.6, 130.4, 144.7, 145.8, 149.1, 152.0; IR 3251, 2969, 2930, 2833, 1585, 1491, $1441,1368,1247,1174,1031 \mathrm{~cm}^{-1} \cdot \lambda_{\max .}=511 \mathrm{~nm}, \log \varepsilon=4.28$. Anal. calcd for $\mathrm{C}_{11} \mathrm{H}_{7} \mathrm{~F}_{4} \mathrm{NO}_{2}: \mathrm{C}$, 50.59; H, 2.70; N, 5.36. Found: C, 50.62; H, 2.74; N, 5.40.

2,3-Diphenyl-5-fluoro-6-methoxy-1,2,3,4-tetrahydroquinoxaline (24'): mp 138 - $139 \quad{ }^{\circ} \mathrm{C}$ $\left(\mathrm{CH}_{3} \mathrm{CN}\right) ;{ }^{1} \mathrm{H}$ NMR $\delta 3.91(\mathrm{~s}, 3 \mathrm{H}), 4.52(\mathrm{~d}, J=7.83 \mathrm{~Hz}, 2 \mathrm{H}), 5.51(\mathrm{br} \mathrm{s}, 2 \mathrm{H}), 6.65-6.87(\mathrm{~m}, 2 \mathrm{H})$, $7.08-7.21(\mathrm{~m}, 8 \mathrm{H}), 7.23-7.26(\mathrm{~m}, 2 \mathrm{H}) ;{ }^{13} \mathrm{C} \mathrm{NMR} \delta 56.5,68.6,104.9,109.7,115.3,121.2,127.1$, 128.3, 128.5, 130.1, 132.3, 138.2, 138.5, 142.4; IR 3350, 3093, 2998, 2952, 1633, 1620, 1591, 1520, $1411,1309,1200,952 \mathrm{~cm}^{-1} . \lambda_{\text {max. }}=413 \mathrm{~nm}, \log \varepsilon=2.49$. Anal. calcd for $\mathrm{C}_{21} \mathrm{H}_{19} \mathrm{FN}_{2} \mathrm{O}: \mathrm{C}, 75.43 ; \mathrm{H}$, 5.73; N, 8.38. Found: C, 75.41; H, 5.74; N, 8.31.

1-Bromo-2-hydroxy-phenylacridine (25): mp $142-144{ }^{\circ} \mathrm{C}\left(\mathrm{CHCl}_{3} / n\right.$-hexane); ${ }^{1} \mathrm{H} \mathrm{NMR} \delta 7.33$ - $7.41(\mathrm{~m}, 4 \mathrm{H}), 7.51-7.53(\mathrm{~m}, 4 \mathrm{H}), 7.65-7.69(\mathrm{~m}, 3 \mathrm{H}) ;{ }^{13} \mathrm{C}$ NMR $\delta 118.6,120.7,125.5,126.3$, $126.9,127.9,128.4,129.0,129.2,129.4,130.1,138.2,148.0,148.5,150.4,157.2$; IR 3300, 2910, 1655, 1557, 1441, 1351, 1260, 1210, 1096, $1043 \mathrm{~cm}^{-1}$. Anal. Calcd for $\mathrm{C}_{19} \mathrm{H}_{12} \mathrm{BrNO}$ : C, 65.16; H, 3.45; N, 4.00. Found: C, 65.21; H, 3.49; N, 3.91. 
5-(3-Bromo-4-hydroxyphenyl)thianthrenium perchlorate (26): mp $202-204{ }^{\circ} \mathrm{C}(\mathrm{MeOH}) ;{ }^{1} \mathrm{H}$

NMR $\delta 6.94-7.11(\mathrm{~m}, 2 \mathrm{H}), 7.59-8.06(\mathrm{~m}, 5 \mathrm{H}), 8.05(\mathrm{~d}, J=7.8 \mathrm{~Hz}, 2 \mathrm{H}), 8.44(\mathrm{~d}, J=7.8 \mathrm{~Hz}, 2 \mathrm{H})$, A OH proton signal is invisible; ${ }^{13} \mathrm{C}$ NMR $\delta 111.9,118.0,118.4,120.7,130.1,130.3,130.6,133.6$, 133.8, 134.9, 135.1, 157.3; IR 3450, 3075, 1593, 1456, 1420, 1150, 1128, 1095, 943, $813 \mathrm{~cm}^{-1}$. Anal. Calcd for $\mathrm{C}_{18} \mathrm{H}_{12} \mathrm{BrClO}_{5} \mathrm{~S}_{2}$ : C, 44.32; H, 2.48; S, 13.15. Found: C, 44.21; H, 2.49; S, 13.22. 